\title{
Controlled Radiolytic Synthesis in the Fluid Stage. Towards Understanding the Effect of the Electron Beam in Liquids
}

Patricia Abellan, ${ }^{123}$ Lucas R. Parent, ${ }^{1}$ Trevor H. Moser, ${ }^{4}$ Chiwoo Park, ${ }^{5}$ Naila Al Hasan, ${ }^{6}$ Prabhakaran Munusamy, ${ }^{7}$ Ivan T. Lucas, ${ }^{8}$ Ilke Arslan, ${ }^{1}$ Jay Grate, ${ }^{1}$ Ayman M. Karim, ${ }^{6}$ James E. Evans, ${ }^{7}$ and Nigel D. Browning. ${ }^{1}$

1. Fundamental \& Comput. Sci. Directorate, Pacific Northwest National Laboratory, Richland, USA.

2. SuperSTEM Laboratory, SciTech Daresbury Campus, Daresbury, UK.

${ }^{3}$ School of Chemical and Process Engineering, University of Leeds, Leeds, UK.

4. Dep. of Mechanical Eng. \& Eng. Mechanics, Michigan Tech. University, Houghton, USA.

5. Dep. of Industrial \& Manufacturing Eng., Florida State University, Tallahassee, USA.

6. Institute for Integrated Catalysis, Pacific Northwest National Laboratory, Richland, USA.

7. Env. Molecular Sci. Laboratory, Pacific Northwest National Laboratory, Richland, USA.

8. UPMC Univ Paris 06, Sorbonne Universities, Paris, France.

Studying liquid samples in the (scanning) transmission electron microscope ((S)TEM) represents specific challenges as compared to the study of crystalline/amorphous solid specimens. Solutions decompose upon $\mathrm{e}^{-}$-beam irradiation through radiolytic processes and chemical species are generated in the liquid phase. These species interact with the sample, most times diffusing away beyond the observation area. For experiments requiring the use of solvents with higher chemical complexity than water, such as organic solvents for battery research and for most synthetic methods, a larger variety of radicals will be produced - with the consequent increase of the number of chemical reactions involved.[1,2] The fundamental understanding of the degradation mechanisms associated with the interaction of the electron beam with different liquid systems is a prerequisite to the accurate description of any dynamic processes. The perfect control of the radiolytic processes using adequate solution mixtures and/or procedures for low dose imaging of liquids in the (S)TEM will expand the range of dynamic processes that can be accurately explored.

In this presentation I will emphasize the importance of understanding and controlling the radiation chemistry of the solvent, which determines the nature and the yields of the reactions involved in the chemical processes induced by the incident electron beam. To illustrate that, I will present our most recent experiments where we achieve an unprecedented control of the amount of the reducing agent involved on the synthesis of well-controlled size $(2.2 \mathrm{~nm})$ palladium particles.[2] I will first detail why some solvents can generate a very low amount of free radicals and therefore enable the precise control of the effect of dose. This is especially important if we take into account the large dose rates typically involved in (S)TEM experiments (within 7-8 orders of magnitude above those used with typical radiation sources [3]). I will also describe particular solvents that can provide one-radical net reduction conditions with no need of added substances for scavenging the oxidizing free radicals. Under such control conditions, we demonstrate a match between the reaction kinetics and reaction products of the radiolytic and chemical syntheses of the size-stabilized Pd nanoparticles. We quantify the effect of electron dose in the STEM on the nucleation kinetics and compare these results to in situ small angle Xray scattering (SAXS) experiments investigating the effect of temperature during chemical synthesis.

Finally, I will present a synthetic method for the preparation of cerium based nanoparticles, i.e. ceria $\left(\mathrm{CeO}_{2}\right)$ and its precursor phase cerium hydroxide III $\left(\mathrm{Ce}(\mathrm{OH})_{3}\right)$, using the effect of the electron beam on 
precursor solutions of $\mathrm{Ce}(\mathrm{III})$ and $\mathrm{Ce}(\mathrm{IV})$ species [4]. In this case we achieve uniform $2.9 \mathrm{~nm} \mathrm{Ce}(\mathrm{OH})_{3}$ particles for their conversion into $\mathrm{CeO}_{2}$. Ceria nanoparticles are particularly interesting because of their redox properties - especially in the 3nm range - which make them great catalysts for a wide range of industrial applications and very promising materials for clinical purposes. In this work, we discuss and interpret the effect of the electron beam on the thermodynamics of cerium, using simplified Pourbaix diagram (E-pH diagram), and the importance of the kinetics of the reactions involved.

Overall, I will introduce methodologies for the precise control of nanoparticle synthesis in the STEM (see images of the final particles in Fig. 1) and provide a mean to uncover the fundamental processes behind the size and shape stabilization of nanoparticles. These methodologies can also be applied to a broader range of experiments requiring the use of complex solvents. The interest of combining results from two different in situ techniques for liquids at the nanoscale, SAXS and STEM will also be emphasized.

\section{References:}

[1] P Abellan et al, Nano Letters 14 (2014) p. 1293

[2] P Abellan et al, "Controlled Radiolytic Synthesis of uniform Palladium nanoparticles", Submitted

[3] N.M. Schneider et al, J. Phys. Chem. C 118 (2014) p. 22373

[4] P Abellan et al, "Radiolytic in situ synthesis of nanometric cerium compounds", Submitted

[5] The work involving development of insitu stages and solutions preparation was supported by the Chemical Imaging Initiative; under the Laboratory Directed Research and Development Program at Pacific Northwest National Laboratory (PNNL). PNNL is a multi-program national laboratory operated by Battelle for the U.S. Department of Energy (DOE) under Contract DE-AC05-76RL01830. A portion of the research was performed using the Environmental Molecular Sciences Laboratory (EMSL), a national scientific user facility sponsored by the Department of Energy's Office of Biological and Environmental Research and located at Pacific Northwest National Laboratory. SuperSTEM is the U.K. National facility for Aberration-Corrected STEM, supported by the Engineering and Physical Research Council (EPSRC).
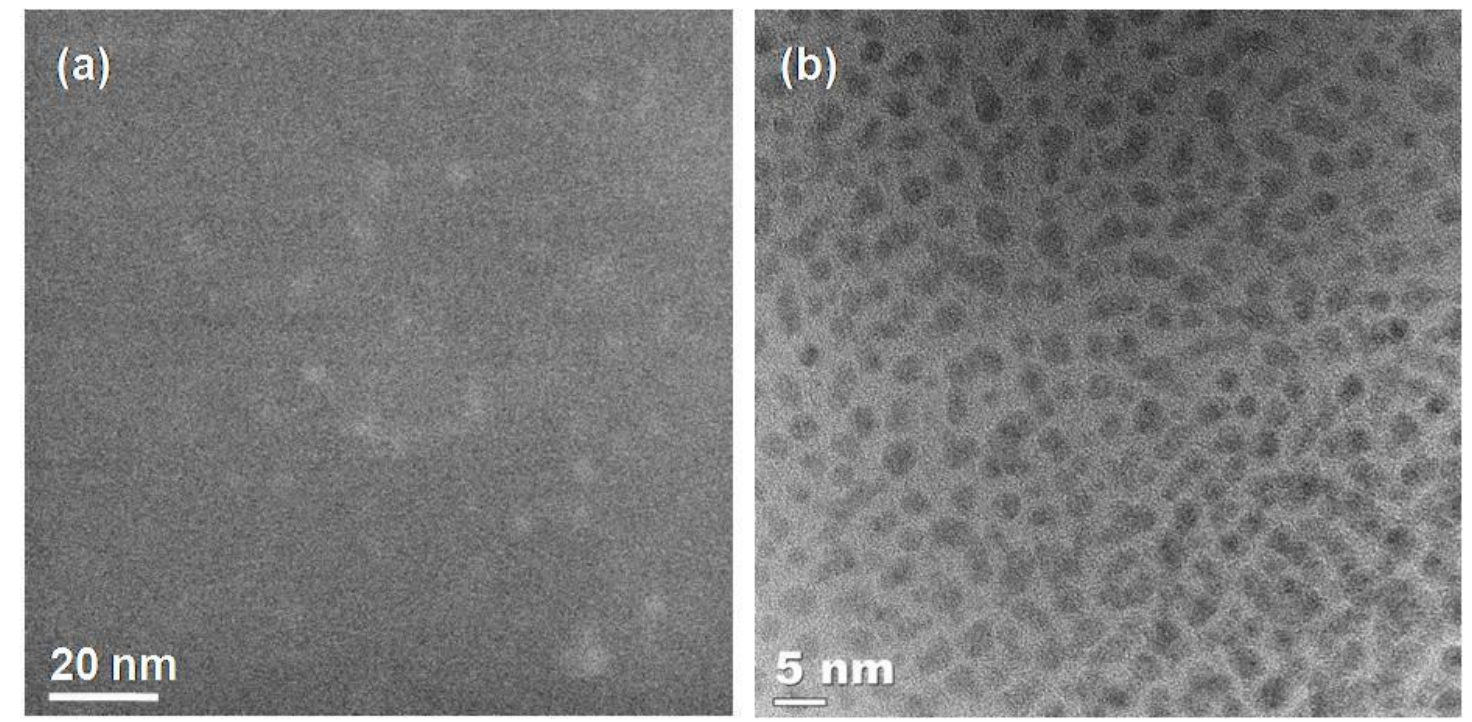

Figure 1. Pd nanoparticles observed through a layer of liquid (a), and cerium hydroxide nanoparticles on a washed chip, (b). Both set of uniform size particles were grown in the fluid stage. 